\title{
Enhancement in pneumatic positioning system using nonlinear gain constrained model predictive controller: experimental validation
}

Siti Fatimah Sulaiman', M. F. Rahmat ${ }^{2}$, Ahmad Athif Faudzi ${ }^{3}$, Khairuddin Osman ${ }^{4}$, N. H. Sunar ${ }^{5}$

${ }^{1,4}$ Advanced Sensors and Embedded Control Research Group, Centre for Telecommunication Research and Innovation (CeTRI), Faculty of Electronics and Computer Engineering, Universiti Teknikal Malaysia Melaka

${ }^{2,3,5}$ School of Electrical Engineering, Universiti Teknologi Malaysia

${ }^{2,3,5}$ Centre for Artificial Intelligence and Robotics (CAIRO), Universiti Teknologi Malaysia

\begin{tabular}{|c|c|}
\hline Article Info & ABSTRACT \\
\hline Article history: & The issues of inaccurate positioning control have made an industrial use of \\
\hline Received May 9, 2021 & $\begin{array}{l}\text { pneumatic actuator remains restricted to certain applications only. Non- } \\
\text { compliance with system limits and properly control the operating system }\end{array}$ \\
\hline Revised Jul 18, 2021 & may also degrade the performance of pneumatic positioning systems. This \\
\hline Accepted Jul 29, 2021 & $\begin{array}{l}\text { study proposed a new approach to enhance pneumatic positioning system } \\
\text { while considering the constraints of system. Firstly, a mathematical model }\end{array}$ \\
\hline Keywords: & $\begin{array}{l}\text { that represented the pneumatic system was determined by system } \\
\text { identification approach. Secondly, model predictive controller (MPC) was }\end{array}$ \\
\hline Pneumatic actuator & developed as a primary controller to control the pneumatic positioning \\
\hline Position control & $\begin{array}{l}\text { system, which took into account the constraints of the system. Next, to } \\
\text { enhance the performance of the overall system, nonlinear gain function was }\end{array}$ \\
\hline Predictive control & incorporated within the MPC algorithm. Finally, the performances were \\
\hline System identification & compared with other control methods such as constrained MPC (CMPC), \\
\hline Transient response & $\begin{array}{l}\text { proportional-integral (PI), and predictive functional control with observer } \\
\text { (PFC-O). The validation based on real-time experimental results for } 100 \mathrm{~mm} \\
\text { positioning control revealed that the incorporation of nonlinear gain within } \\
\text { the MPC algorithm improved } 21.03 \% \text { and } 2.69 \% \text { of the speed response given } \\
\text { by CMPC and PFC-O, and reduced } 100 \% \text { of the overshoot given by CMPC } \\
\text { and PI controller; thus, providing fast and accurate pneumatic positioning } \\
\text { control system. }\end{array}$ \\
\hline
\end{tabular}

This is an open access article under the CC BY-SA license.

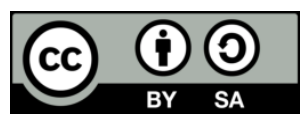

\section{Corresponding Author:}

Siti Fatimah Sulaiman

Centre for Telecommunication Research and Innovation (CeTRI)

Faculty of Electronics and Computer Engineering

Universiti Teknikal Malaysia Melaka

76100 Durian Tunggal, Melaka, Malaysia

Email: sitifatimahsulaiman@utem.edu.my

\section{INTRODUCTION}

The pneumatic system is often associated with the issues of nonlinearities and uncertainties such as compressibility of air and friction effect surrounding the system [1], [2]. Therefore, it is a challenging task to control the system for a certain desired performance. Controlling the system's position also becomes more complicated with the need to simultaneously consider the accuracy of system and response time. Various controllers were reportedly proposed to control the position of pneumatic system; such as proportionalintegral-derivative (PID) [3], pole-placement [4], Ho loop shaping controller [5], adaptive controller [6], 
fuzzy logic [7], and predictive control [8], [9]. However, most of these reported controllers were incapable to simultaneously deliver high-speed response with accuracy. It is also crucial to address the constraints of the system in the design of controller for the actual applications of the system. The non-compliance with the prescribed constraints may cause damage to the pneumatic system and its components and affects the overall performance of the control system itself. Hence, this study regarded the significance of considering the constraints of the pneumatic system during the development of the controller.

This study proposed the model predictive controller (MPC) as the control strategy for the positioning of the system. MPC can considers the constraints, deals with multivariable control issue, and also suitable for single-input and single-output (SISO) system and multi-input and multi-output (MIMO) system [10]-[12], which explain why it is highly favourable in the process industries. MPC is also reported used to control robots [13], [14]. Figure 1 shows the basic structure of MPC.

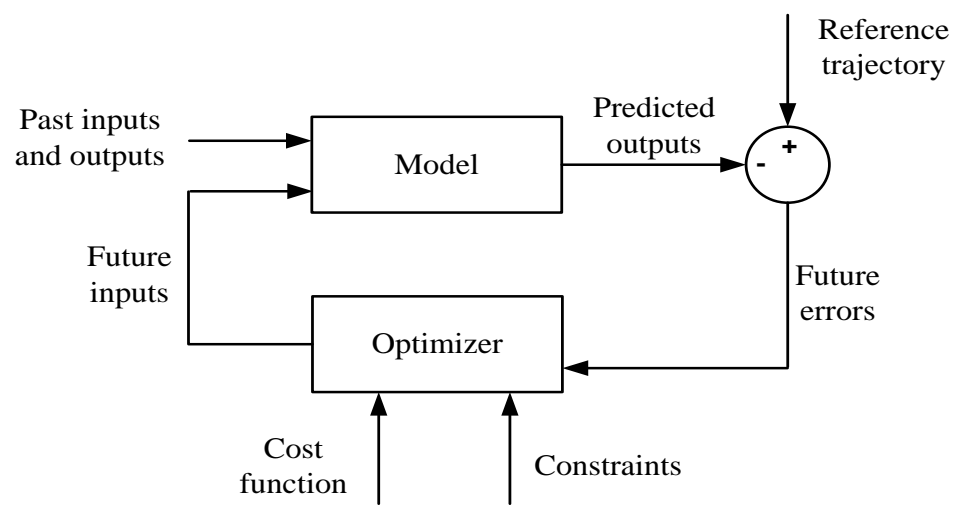

Figure 1. Basic structure of MPC [11]

MPC in Figure 1 uses a process model to predict the future output based on the previous and current values by solving the optimal future control actions at each sampling time instant [11]. The optimizer considers the cost function and the constraints of the system to calculate the control actions. In particular, the added constraints in MPC prevent the wind-up phenomenon. With the use of MPC as a strategy to control the position of the pneumatic system used in this study, the constraints in the pneumatic system can be considered [15].

The employment of MPC as a control strategy to control the position of pneumatic system used in this study has been evaluated in previous studies [16], [17]. In order to verify the effectiveness of the strategy in handling systems with constraints, constraints were applied to the input of the system (control signal to the valves). From the study, they found that constrained MPC successfully produced accurate tracking compared to unconstrained MPC [16], [17]. However, they found that the inclusion of constraints in the MPC algorithm reduced the system response and aggressiveness of the pneumatic system since it requires more computational effort to optimise the cost function (compared to the unconstrained case) [16], [17]. These findings were consistent with several previous studies [11], which demonstrated that the inclusion of constraints in the MPC algorithm did reduce the response time in the system output. Thus, the initial use of constrained MPC was incapable to provide accurate and timely positioning response of pneumatic system used in this study [16], [17]. The use of linear controller to control a system, especially the nonlinear system, is typically also incapable to simultaneously deliver high-speed response with accuracy [18]. Despite that, this study considered that the system can be improved through certain modifications.

This study focused on the approach adopted by Seraji [19], considering that its implementation is effective and straightforward. Seraji [19] improved the conventional PID controller by combining a nonlinear gain in cascade and a linear fixed-gain PID controller in order to control the robotic arm. Consequently, the controller is able to adapt its response based on the performance of the closed-loop control system. When the error of the controlled variable is large, the gain amplifies the error to generate a large corrective action to rapidly drive the system output, resulting in high-speed response. When the error diminishes, the gain is automatically reduced to prevent excessive oscillations and large overshoots in the response, resulting in accurate response with zero steady-state error. In other words, the controller possesses the advantage of high initial gain (which delivers high-speed response) and low gain (that prevents an oscillatory behaviour in the system response given its automatic nonlinear gain adjustment). In another study, Rahmat et al. [20] found 
that the combination of a nonlinear gain and the PID controller reduces the overshoot and produces accurate tracking in the pneumatic positioning system response, which was corroborated by Salim et al. [21]. The study also demonstrated the enhancement of pneumatic positioning system in the transient response of the system where the nonlinear gain function and PID controller were combined to control the cylinder stroke of pneumatic system with a payload of maximum weight up to $28 \mathrm{~kg}$ at the end of the pneumatic cylinder stroke. The effectiveness of the proposed approach by Seraji [19] in reducing the overshoot and producing better tracking in the system performance was proven using various applications, such as in robotics, milling systems, and wastewater treatment process [22]-[24].

Given such considerations, this study incorporated a nonlinear gain in the conventional constrained MPC (CMPC) as a new control strategy to improve speed response and accuracy of the pneumatic positioning system. Since this study involves with real-time implementation, therefore, this study also considered the use of observer in the design of control strategy. Essentially, observer is used to estimate the internal state variable of the real system [25]. This study is divided into 6 sections. The background of the study is discussed in section 1. Section 2 provides the information about the pneumatic system employed in this study by describing the system's components and explaining its operation. Section 3 explains the experimental setup and the process of modelling the system using a system identification technique. The procedures in designing the proposed control strategy to perform the control task are presented in section 4. Section 5 presents and discusses the experimental results of the proposed controller. The effectiveness of the proposed control strategy in comparison to the other controllers is also demonstrated in section 5 and lastly, section 6 concludes the overall findings of the study.

\section{PNEUMATIC SYSTEM DESCRIPTION}

The pneumatic system used in this study is shown in Figure 2. It was equipped with optical sensor (AEDR-8300), laser stripe rod, pressure sensor (KOGANEI: PSU-EM-S)), valves (KOGANEI: EB10ES1PS-6W), and programmable system on chip (PSoC) control board. The pneumatic system used in this study is a double-acting type cylinder (KOGANEI-HA: twinport cylinders) with $16 \mathrm{~mm}$ rod diameter and $200 \mathrm{~mm}$ rod stroke length. The schematic diagram of the system is illustrated in Figure 3.

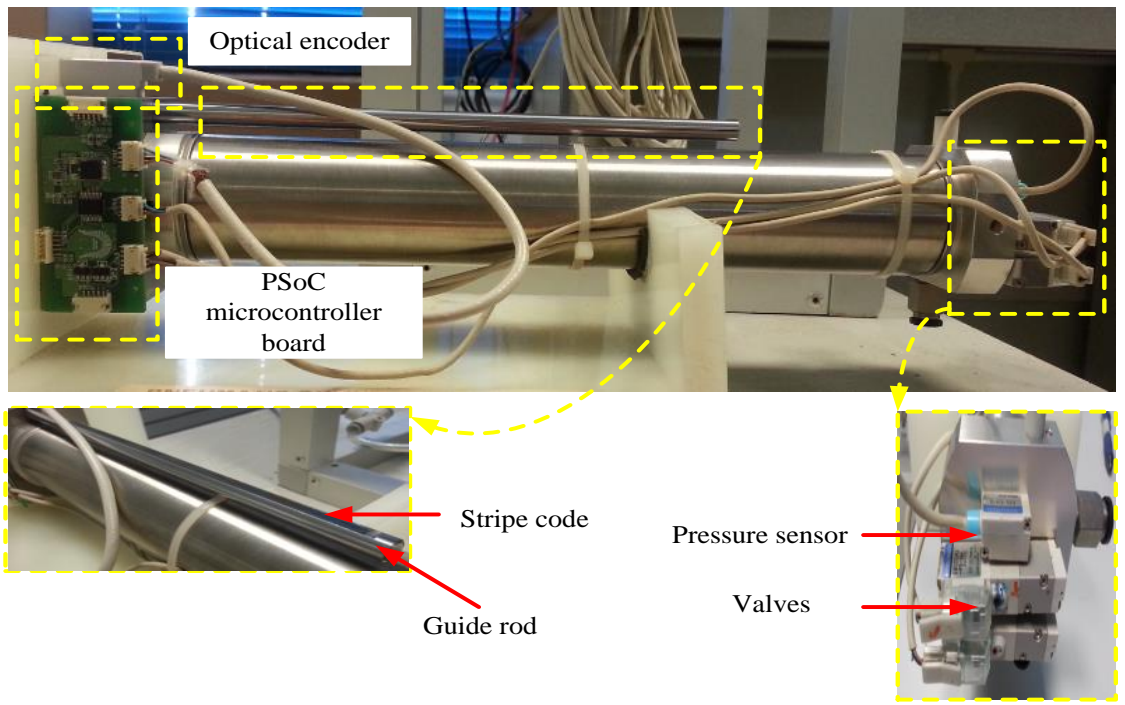

Figure 2. The pneumatic system and its main components

Two valves which were attached at the end of the cylinder, were employed to control the inlet and outlet air of the cylinder. In this study, the extension and retraction of the cylinder stroke are manipulated by the duty cycle of a pulse-width modulator (PWM) signal to drive the valves. The PWM model in this study has a precision of 8-bit. When the PWM model receives a positive signal from the plant or controller, it will convert the signal into equivalent PWM signal and send that signal to the valve 1 to perform extension. If the PWM model receives negative signal, the model will send the signal to valve 2 to perform retraction. 


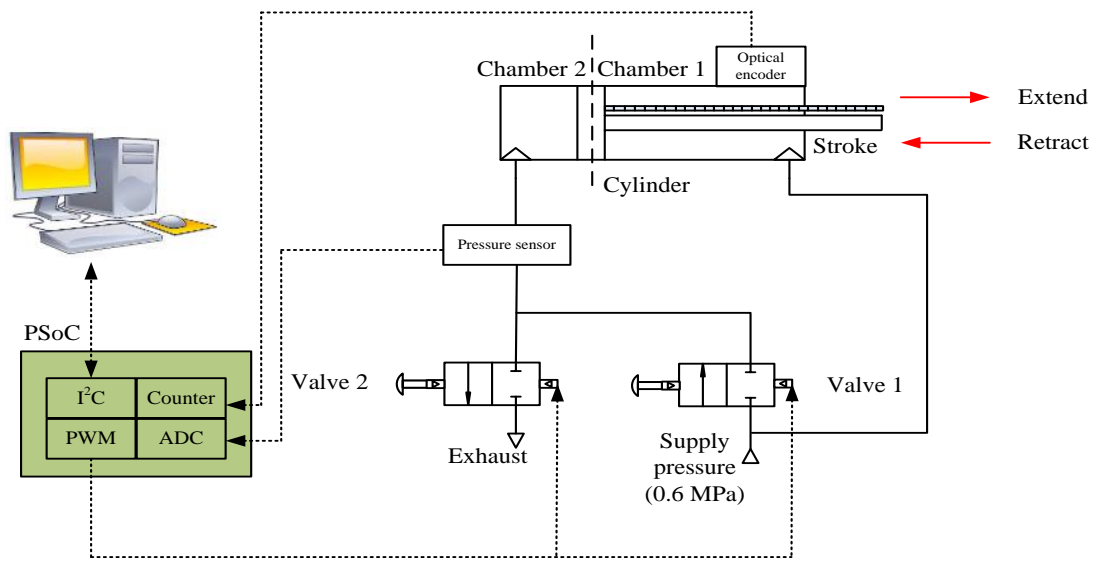

Figure 3. The pneumatic system schematic diagram

\section{MODEL IDENTIFICATION}

Figure 4 shows the experimental set-up, as a part of the process to obtain the mathematical model of the system. In this study, a mathematical model of the pneumatic system was identified by means of system identification. The platform for this study was MATLAB/Simulink, which was equipped in the computer. The nominal pressure used was 0.6 MPa and a national instrument (NI) (PCI/PXI-6221) was used for data acquisition (DAQ) system.

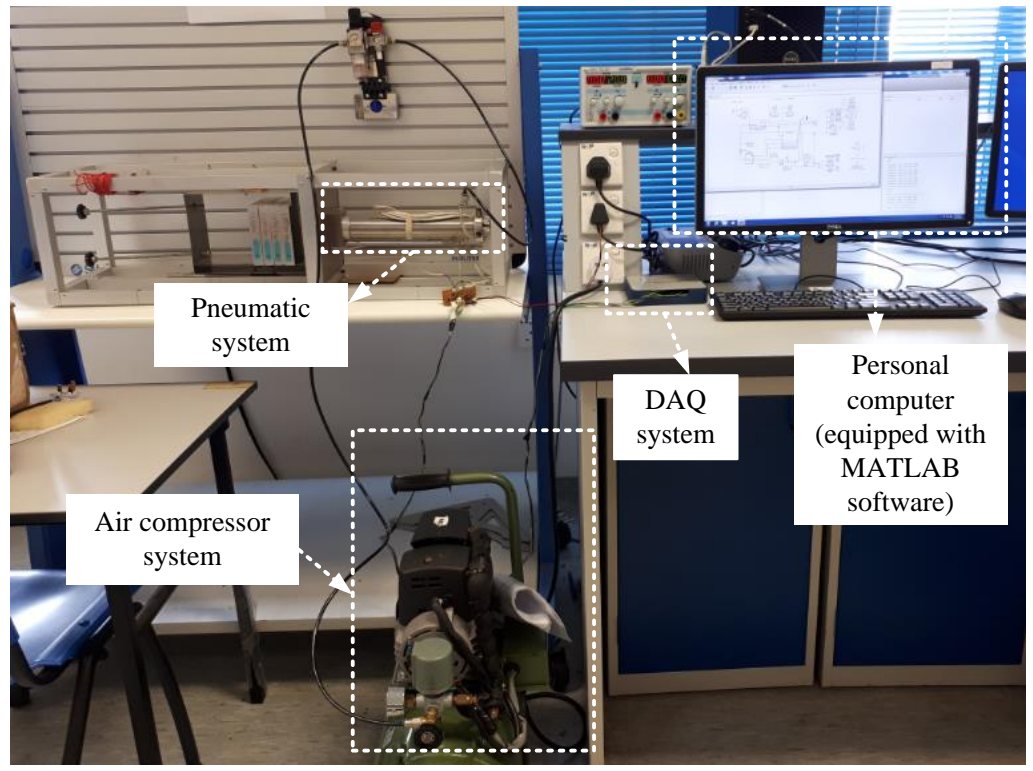

Figure 4. The set-up of experiment

A total of 2000 measurements of input and output data were collected at sampling time $\left(T_{s}\right)$ of 10 ms during experiment. The input data contains 2000 data points of continuous step signal applied to the valves, while the output data consist of 2000 measurements of cylinder stroke position signal. The AutoRegressive with eXogenous input (ARX) parametric model was chosen for this study since it satisfies the criteria for system identification. The identified discrete state-space model based ARX model structure utilized throughout this study is represented by (1).

$$
A_{p}=\left[\begin{array}{ccc}
0 & 1 & 0 \\
0 & 0 & 1 \\
0.1284 & -0.9976 & 1.8690
\end{array}\right], B_{p}=\left[\begin{array}{l}
0 \\
0 \\
1
\end{array}\right], C_{p}=\left[\begin{array}{lll}
0.0016 & 0 & 0
\end{array}\right], D_{p}=[0]
$$


The identified model fits the actual plant model at a value of approximately $91.09 \%$. The loss of $8.91 \%$ may be due to dead-zone, friction and air leakage. in the pneumatic system itself. All the nonlinearities in this study are neglected and the system utilized is assumed to be a linear system. As the model in (1) provides all the poles inside the unit circle $(0.1887,0.6811$ and 0.9992$)$, it is considered stable.

\section{CONTROLLER DESIGN}

This section discusses the relevant design of the conventional constrained MPC (CMPC) to control the position of pneumatic system with certain modifications for enhanced performance.

\subsection{MPC formulation}

MPC is a type of controller that is designed based on a mathematical model of the plant. In this study, the pneumatic model used was taken to be a state-space model, and the pneumatic system used in this study was assumed as a SISO system. The deterministic model of pneumatic system employed in the study with $p$ inputs and $q$ outputs is described as (2) and (3).

$$
\begin{aligned}
& x_{p}(k+1)=A_{p} x_{p}(k)+B_{p} u(k) \\
& y(k)=C_{p} x_{p}(k)+D_{p} u(k)
\end{aligned}
$$

Where $A_{p}, B_{p}, C_{p}$, and $D_{p}$ are system matrices with appropriate dimensions, $x_{p}$ is the state variable vector with dimension $n, u$ is the input variable vector, and $y$ is the process output vector. In this study, $p$ and $q=1$ since the system is a SISO system. Matrix $D_{p}$ is assumed to be zero in order to demonstrate that there is no direct feed through the input, $u(k)$ and the output, $y(k)$. This is due to the principle of receding horizon control itself, in which only a current information of the plant is required for prediction and control. The model in (2) was improved in order to include an integrator in the design. The different on both sides of (2) yielded the incremental state-space in (4),

$$
\Delta x_{p}(k+1)=A_{p} \Delta x_{p}(k)+B_{p} \Delta u(k)
$$

Where,

$$
\Delta x_{p}(k+1)=x_{p}(k+1)-x_{p}(k), \Delta x_{p}(k)=x_{p}(k)-x_{p}(k-1), \Delta u(k)=u(k)-u(k-1)
$$

$\Delta x_{p}(k+1)$ and $\Delta x_{p}(k)$ denote the difference of the state variable, and $\Delta u(k)$ denotes the difference of the control variable. A new state variable vector in (5) was selected in order to connect the state variable $\Delta x_{p}(k)$ to the output $y(k)$.

$$
x(k)=\left[\begin{array}{ll}
\Delta x_{p}(k)^{T} & y(k)
\end{array}\right]^{T}
$$

Considering (4) in output, (3) can be written in the form of (6),

$$
y(k+1)-y(k)=C_{p}\left(x_{p}(k+1)-x_{p}(k)\right)
$$

Rearrange (6) based on (4) gives,

$$
y(k+1)=C_{p} A_{p} \Delta x_{p}(k)+C_{p} B_{p} \Delta u(k)+y(k)
$$

The augmented state-space model as indicated in (8) can be obtained by putting together (4) and (7).

$$
x(k+1)=A x(k)+B \Delta u(k), y(k)=C x(k)
$$

Where

$$
A=\left[\begin{array}{cc}
A_{p} & 0_{p}{ }^{T} \\
C_{p} A_{p} & I
\end{array}\right], B=\left[\begin{array}{c}
B_{p} \\
C_{p} B_{p}
\end{array}\right], C=\left[\begin{array}{ll}
0_{p} & 1
\end{array}\right]
$$

$A, B$ and $C$ is the augmented model, $0_{p}$ is the zero matrix with dimension $q \times n$ and $I$ is a unit matrix with dimension $q \times q$. Considering (1) as a plant model, the augmented model of the pneumatic system utilized in this study is represented as, 


$$
A=\left[\begin{array}{cccc}
0 & 1 & 0 & 0 \\
0 & 0 & 1 & 0 \\
0.1284 & -0.9976 & 1.869 & 0 \\
0 & 0.0016 & 0 & 1
\end{array}\right], B=\left[\begin{array}{l}
0 \\
0 \\
1 \\
0
\end{array}\right], C=\left[\begin{array}{llll}
0 & 0 & 0 & 1
\end{array}\right]
$$

The augmented model in (9) has eigenvalues $\lambda$ at $1,0.9992,06811$, and 0.1887 . The first $\lambda$ is from the augmentation of the plant model, while the last three $\lambda$ are from the original pneumatic plant. Consequently, the augmented state-space model has one integrator embedded into the augmented state-space model to suit the controller design purpose. The design of MPC is based on optimizing the difference of the control signal $\Delta u(k)$ within an optimization window. $\Delta u(k)$, or so called the future control trajectory is denoted by (10),

$$
\Delta u(k), \Delta u(k+1), \cdots, \Delta u\left(k+N_{c}-1\right)
$$

Where $N_{c}$ is the control horizon, which is used to dictate the number of parameters in order to capture the future control trajectory. When the state variable vector $x(k)$ at sampling time instant $k$ is assumed available through measurement, the current plant information is provided by the state $x(k)$. With given information $x(k)$, the future state variables are denoted as (11),

$$
x(k+1 \mid k), x(k+2 \mid k), \cdots, x(k+m \mid k), \cdots, x\left(k+N_{p} \mid k\right)
$$

Where $x(k+m \mid k)$ is the predicted state variable at $k+m$ with the given current plant information $x(k)$ and $N_{p}$ is the prediction horizon or the length of optimization window. Generally, $N_{c} \leq N_{p}$. In this study, $N_{c}$ and $N_{p}$ were chosen to be 3 and 20, respectively. The sequentially calculated future state variable using the set of future control parameters is expressed,

$$
\begin{aligned}
& x(k+1 \mid k)=A x(k)+B \Delta u(k) \\
& x(k+2 \mid k)=A^{2} x(k)+A B \Delta u(k)+B \Delta u(k+1) \\
& x\left(k+N_{p} \mid k\right)=A^{N_{p}} x(k)+A^{N_{p}-1} B \Delta u(k)+A^{N_{p}-2} B \Delta u(k+1)+\cdots \\
& +A^{N_{p}-N_{c}} B \Delta u\left(k+N_{c}-1\right)
\end{aligned}
$$

The substitution of (12) into output in (8) provides the predicted output variables, as shown in (13),

$$
\begin{aligned}
& y(k+1 \mid k)=C A x(k)+C B \Delta u(k) \\
& y(k+2 \mid k)=C A^{2} x(k)+C A B \Delta u(k)+C B \Delta u(k+1) \\
& y\left(k+N_{p} \mid k\right)=C A^{N_{p}} x(k)+C A^{N_{p}-1} B \Delta u(k)+C A^{N_{p}-2} B \Delta u(k+1)+\cdots \\
& +C A^{N_{p}-N_{c}} B \Delta u\left(k+N_{c}-1\right)
\end{aligned}
$$

Besides, (13) can also be written in a compact matrix form as,

$$
Y=F x(k)+\Phi \Delta U
$$

Where,

$$
F=\left[\begin{array}{c}
\mathrm{CA} \\
\mathrm{CA}^{2} \\
\vdots \\
C A^{N_{p}}
\end{array}\right], \Phi=\left[\begin{array}{cccc}
C B & 0 & \cdots & 0 \\
C A B & C B & \cdots & 0 \\
\vdots & \vdots & \vdots & \vdots \\
C A^{N_{p}-1} B & C A^{N_{p}-2} B & \cdots & C A^{N_{p}-N_{C}} B
\end{array}\right]
$$

In this study, a state estimator or an observer system was employed in the design of control strategy. Assuming at time $k$, the information of state variable $x(k)$ was not measurable (or available), an observer system will be used to estimate the state variable $x(k)$ from the process measurement. Hence, the future state variable in (12) was calculated using the estimated state variables as in (15),

$$
\hat{x}(k+1)=A \hat{x}(k)+B \Delta u(k)+K_{o b s}(y(k)-C \hat{x}(k))
$$


Where $K_{o b s}$ is the gain matrix. The pole-placement was employed as a technique to calculate the suitable $K_{o b s}$ value. The poles, which were used to find the suitable $K_{o b s}$ are to be were maintained in the unit circle in order to ensure the stability of the system. The value of poles assigned in this study is demonstrated in Table 1. In this study, the sequence of $\Delta u(k), \Delta u(k+1), \cdots, \Delta u\left(k+N_{c}-1\right)$ in (10) was approximated using a set of discrete-time Laguerre functions. The set of discrete-time Laguerre functions expressed a vector form described in (16), while (17) expressed its difference equation,

$$
\begin{aligned}
& L(k)=\left[\begin{array}{llll}
l_{1}(k) & l_{2}(k) & \cdots & l_{N}(k)
\end{array}\right]^{T} \\
& L(k+1)=A_{l} L(k)
\end{aligned}
$$

Where $A_{l}$ is the matrix with dimension $N \times N$ and is a function of parameters $\alpha$ and $\beta$. The initial condition of (16) is given in,

$$
L(0)=\sqrt{\beta} \times\left[\begin{array}{lllll}
1 & -\alpha & \alpha^{2} & \cdots & (-1)^{N-1} \alpha^{N-1}
\end{array}\right]^{T}
$$

$\alpha$ is the scaling factor of the Laguerre network and $\beta=1-\alpha^{2}$. To ensure the stability of the network, $\alpha$ must be within $0 \leq \alpha<1$. In this study, the value of $\alpha$ used is 0.1 . This study is set out to respect the actuator constraints while bringing and maintaining the position of the cylinder stroke as close as possible to the desired position. In doing so, the manipulated and controlled variables being considered were the signal to the valves and the position of the cylinder stroke. MPC was used in this study to determine the future adjustments of the signal to the valve. MPC predicted the future plant outputs and performed the control actions accordingly by solving the optimal future control actions (cost function and constraints). The cost function $J$ that reflect the control objective of this study is defined as,

$$
J=\sum_{m=1}^{N_{p}} x(k+m \mid k)^{T} Q x(k+m \mid k)+\eta^{T} R \eta
$$

Where $Q$ and $R$ are the weighting matrices with $Q=C^{T} C$ (or $\geq 0$ ) and $R$ was chosen to be 0.1 (or $>0$ ).

$$
Q=C^{T} C=\left[\begin{array}{llll}
0 & 0 & 0 & 0 \\
0 & 0 & 0 & 0 \\
0 & 0 & 0 & 0 \\
0 & 0 & 0 & 1
\end{array}\right]
$$

Where,

$$
C=\left[\begin{array}{llll}
0 & 0 & 0 & 1
\end{array}\right]
$$

Since this study aims to bring and maintain the predicted output as close as possible to the set-point signal, the state variable $x(k+m \mid k)$ in (19) must be re-defined in order to include the set-point signal in the cost function equation. Hence, $(21)$ is the new equation of the state variable with the inclusion of set-point signal $r(k)$ within the optimization window,

$$
x(k+m \mid k)=\left[\Delta x_{p}(k+m \mid k)^{T} \quad y(k+m \mid k)-r(k)\right]^{T}
$$

The optimal solution of the parameter vector $\eta$ in (19) based on minimization of the cost function is represented as (22),

$$
\eta=-\Omega^{-1} \psi x(k)
$$

Where,

$$
\Omega=\sum_{m=1}^{N_{p}} \phi(m) Q \phi(m)^{T}+R, \psi=\sum_{m=1}^{N_{p}} \phi(m) Q A^{m}
$$

Upon obtaining the optimal solution of the parameter vector $\eta$, the control law can is realised as,

$$
\Delta u(k)=L(0)^{T} \eta
$$

The control law in (23) can also be represented in the form of linear state feedback control as expressed in (24),

Enhancement in pneumatic positioning system using nonlinear gain constrained... (Siti Fatimah Sulaiman) 


$$
\Delta u(k)=-K_{m p c} x(k)
$$

Where,

$$
K_{m p c}=L(0)^{T} \Omega^{-1} \psi, x(k)=\left[\begin{array}{ll}
\Delta x_{p}(k)^{T} & e(k)
\end{array}\right]^{T}
$$

Hence, the control law to be applied can be written as,

$$
u(k)=\Delta u(k)+u(k-1)
$$

$e(k)$ is the error signal between the predicted output $y(k)$ and set-point signal $r(k)$. When an observer is used in the design, all the actual state variable $x(k)$ will be replaced with the observed state variable $\hat{x}(k)$.

\subsection{MPC with constraints on input valves}

Restrictions were given to the control signal to the system's manipulated variable or input valves in the case of the pneumatic system used in this study. In this study, a control signal was defined as a signal exported from the controller, which will influence the system response (i.e. the position of the cylinder stroke). Thus, this signal should be controlled to ensure that would always be in a range that is allowed by the system. If the maximum allowable value is exceeded, an overshoot might be generated in the system response. This phenomenon may occur frequently as the system is implemented in real-time environment. The maximum amplitude value allowed for the extension and retraction of the cylinder stroke during operation were set to +255 (for valve 1) and -255 (for valve 2), respectively. Hence, the signal from the MPC to the system's valves was constrained within \pm 255 . Suppose that the limits on the valves control signals are $u_{\min }=-255$ and $u_{\max }=+255$, and $u(k)=\sum_{i=0}^{k-1} \Delta u(k)$. Consequently, the inequality constraints for future time $k, k=1,2, \ldots$ can be expressed as:

$$
-255 \leq M \eta+u(k-1) \leq+255
$$

Where,

$$
M=\left[\begin{array}{cccc}
\sum_{i=0}^{k-1} L_{1}(i)^{T} & 0_{2}{ }^{T} & \cdots & 0_{m}{ }^{T} \\
0_{1}{ }^{T} & \sum_{i=0}^{k-1} L_{2}(i)^{T} & \cdots & 0_{m}{ }^{T} \\
\vdots & \vdots & \vdots & \vdots \\
0_{1}{ }^{T} & 0_{2}{ }^{T} & \cdots & \sum_{i=0}^{k-1} L_{m}(i)^{T}
\end{array}\right]
$$

Wwhere $u(k-1)$ is the previous control signal and $0_{k}{ }^{T}$ is a row vector with dimension as in $L_{k}(0)^{T}$.

\subsection{The design of nonlinear gain constrained model predictive controller}

This study incorporated the nonlinear gain in a controller algorithm known as the constrained model predictive controller (CMPC) as a new approach to enhance the performance of pneumatic positioning system, especially in its transient response. The justifications of incorporating the nonlinear gain within the controller algorithm as a new approach for this study included the following: 1) it is challenging to provide good transient response with the use of linear controller (which in this case, the conventional constrained MPC) for the system (in other words, it is impossible to achieve high speed response without overshoot with the use of linear controller, particularly in real-time environment) due to the existence of nonlinearities and uncertainties in the system and 2) the inclusion of constraints in the controller algorithm degraded the response time of the system; thus, making the system slower.

This study also considered that the use of linear controller remains relevant in controlling the position of the pneumatic system in this study and certain modifications can improve the performance of system. Thus, the underlying principle to this proposed control strategy for this study was to employ nonlinear elements in the linear controller scheme, which compensate for error variations in the pneumatic positioning system, while simultaneously improving the performance of system. Figure 5 illustrates the block diagram of the pneumatic positioning system with the proposed control strategy.

As shown in Figure 5, a function called nonlinear gain was employed in this study to compensate the nonlinearities and uncertainties in the system parameters. It was utilized to control the error signal $e(k)$ between the predicted output $y(k)$ and set-point signal $r(k)$ in (24). It is of utmost importance to control $e(k)$ due to its influence on the formation of the control signal to the pneumatic system. This technique was employed in this study to adjust the controller gain according to the output produced from this function, which is known as the scaled error $f(e)$ as described in (27). 


$$
f(e)=k_{n l}(e) \times e(k)
$$

Where,

$$
\begin{aligned}
& k_{n l}(e)=\frac{\exp (k e)+\exp (-k e)}{2} \\
& e=\left\{\begin{array}{cl}
e & |e| \leq e_{\text {max }} \\
e_{\max } \times \operatorname{sign}(e) & |e|>e_{\text {max }}
\end{array}\right.
\end{aligned}
$$

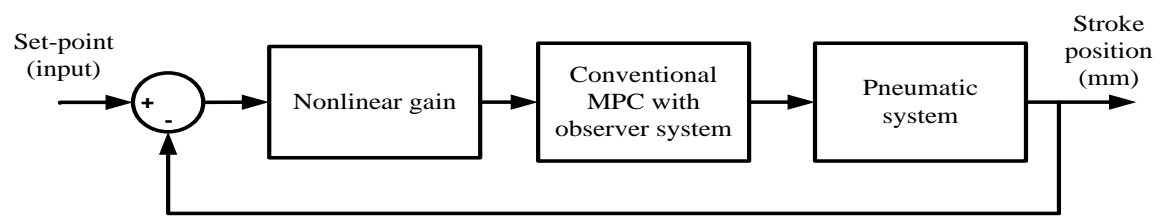

Figure 5. Block diagram of the proposed control strategy

The value of nonlinear gain function $k_{n l}(e)$ in (28) was adjusted according to the error $e$ from the system, while the parameter values of both rate variation of nonlinear gain $(k)$ and variation of error $\left(e_{\max }\right)$ were selected by the user. $k_{n l}(e)$ acts as a nonlinear function of error $e$ and is bounded in the sector as described in (29). Figure 6 illustrates the relationship between $k_{n l}$ and $e$.

$$
0 \leq k_{n l}(e) \leq k_{n l}\left(e_{\max }\right)
$$

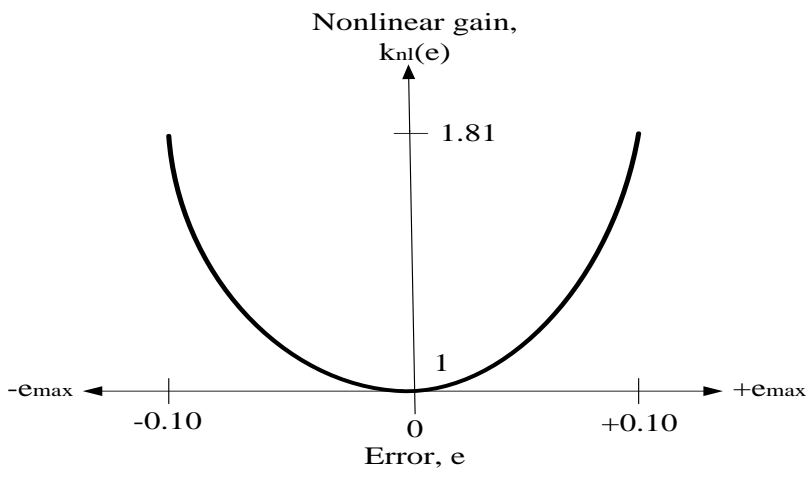

Figure 6. Relationship between $k_{n l}$ and $e$

The incorporation of a nonlinear gain function into the controller algorithm in (25) generates the proposed control strategy (30). This study performed the following procedures in Figure 7 to determine the parameter values of $k_{n l}$.

$$
\begin{aligned}
& u(k)=-\left[K_{m p c 1} \Delta x_{1}(k)+K_{m p c 2} \Delta x_{2}(k)+K_{m p c 3} \Delta x_{3}(k)+K_{m p c 4}\left(k_{n l}(e) \times e(k)\right)\right] \\
& +u(k-1)
\end{aligned}
$$

As shown in Figure 7, prior to the selection of the value of parameters $k$ and $e_{\max }(28)$, the maximum value of $k_{n l}$ for stability must be obtained. It was revealed in the closed-loop stability using Jury stability test that the performance of the proposed control system tend to unstable when $k_{n l} \geq 2.96$. Thus, it is recommended that $k_{n l}$ is within the range of $0<k_{n l}<2.693$ for system stability. Based on the prior procedures and tests, the recommended value of $k$ and $e_{\max }$ were set at 12 and 0.1 , respectively. Table 1 describes the parameters of the proposed control strategy used in this study.

Based on (28), when $k=12$ and $e_{\max }=0.1, k_{n l}\left(e_{\max }\right)=1.811$, which implies that it is within the stable region. Generally, higher value of $e_{\max }$ contributes the highest overshoot, which leads to

Enhancement in pneumatic positioning system using nonlinear gain constrained... (Siti Fatimah Sulaiman) 
uncontrollable and unstable response in the system. In addition, utilisation of this technique automatically adjusts the value of $k_{n l}$ according to the generated value of $e(k)$ at each time instant. When there is no error present, $k_{n l}(e)=1$. In other words, the controller system reacts similarly to other conventional MPC without the presence of $k_{n l}$ in the controller system. However, with the presence of error in the system and inclusion of $k_{n l}$, the controller signal will be adjusted accordingly to the value of $k_{n l}(e)$. The key advantage of this technique is the fact the controller gain value, expect for the values of $k$ and $e_{\text {max }}$, does not require tuning.

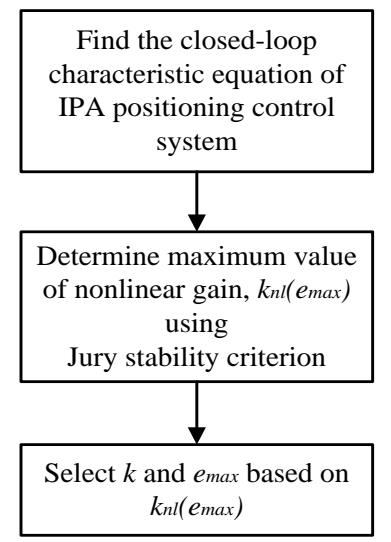

Figure 7. Procedures to determine the parameter values of nonlinear gain $\left(k_{n l}\right)$

Table 1. Description of the controller's parameters

\begin{tabular}{llcc}
\hline \multicolumn{1}{c}{ Control strategies } & \multicolumn{1}{c}{ Name of parameter } & $\begin{array}{c}\text { Control parameters } \\
\text { Abbreviation }\end{array}$ & Value \\
\hline \multirow{4}{*}{ MPC } & Prediction horizon & $N_{p}$ & 20 \\
& Control horizon & $N_{c}$ & 3 \\
Observer & Scaling factor of Laguerre network & $\alpha$ & 0.1 \\
Nonlinear gain function & $R$ & 0.1 \\
& Weighting matrix & - & $0.0100,0.0105,0.0110,0.0115$ \\
& Observer poles & $k$ & 12 \\
& Variation of nonlinear gain & $e_{\max }$ & 0.1 \\
\hline
\end{tabular}

\section{RESULTS AND DISCUSSION}

The capabilities of the proposed control strategy (NG-CMPC) to control and to improve the transient response performance of the pneumatic positioning system, in a real-time environment are evaluated and discussed in this section. The positioning control performance for different distances using the proposed control strategy (NG-CMPC) was presented and evaluated. The performances of the proposed control strategy were also analysed and compared to the existing methods of similar pneumatic plant systems (refer to pneumatic system used in this study) in order to determine the improvements. A sampling time of $10 \mathrm{~ms}$ was used in experimenting, and the proposed control strategy was developed using MATLAB/Simulink. The controller parameters stated in Table 1 was used in this section in order to perform validation. In this subsection, experiments were conducted to test the implementation of the proposed control strategy (NG-CMPC) in order to control thepneumatic positioning system at different distances. Several values of position distance $(50 \mathrm{~mm}, 100 \mathrm{~mm}$, and $150 \mathrm{~mm}$ ) were provided for comparison, and the step signal was applied as the input signal. Each test was conducted for $20 \mathrm{~s}$. The performances of the NG-CMPC system's transient (i.e. rise time $\left(t_{r}\right)$, settling time $\left(t_{s}\right)$, overshoot $(O S)$, and steady-state error $\left(e_{s S}\right)$ in controlling the pneumatic positioning systems for all distances were then compared with CMPC, PFC-O, and PI. Figure 8 shows a comparative view of these step tests where the position distance was varied from fully retracted $(0 \mathrm{~mm})$ to near fully retracted $(50 \mathrm{~mm})$, fully retracted $(0 \mathrm{~mm})$ to the actuator mid-stroke $(100 \mathrm{~mm})$, and fully retracted $(0 \mathrm{~mm})$ to near fully extended $(150 \mathrm{~mm})$. Table 2 summarizes the data obtained in Figure 8.

The experimental results in Figure 8 and Table 2 demonstrate an increase in the response time of $t_{r}$ and $t_{s}$ for all strategies as the position distance is increased. In other words, the longer the positioning distance to be reached by the cylinder stroke, the longer the time taken by all strategies to achieve its steadystate value. The comparison between the controller strategies shows that NG-CMPC successfully controlled the pneumatic cylinder stroke the fastest in order to achieve a positioning distance of $50 \mathrm{~mm}, 100 \mathrm{~mm}$, and 
$150 \mathrm{~mm}$. The speed response generated by PFC-O for all the distances was almost similar to the performance provided by NG-CMPC, with some additional time. Among all, the PI controller took a longer time to be within $2 \%$ of the steady-state value. The PI controller took about $3.500 \mathrm{~s}, 3.345 \mathrm{~s}$, and $3.826 \mathrm{~s}$ to be within $\pm 2 \%$ of the position of $50 \mathrm{~mm}, 100 \mathrm{~mm}$, and $150 \mathrm{~mm}$ respectively. The observation through the data in Table 2 found that NG-CMPC successfully improved the overall performance of $t_{r}$ and $t_{s}$ produced by CMPC.

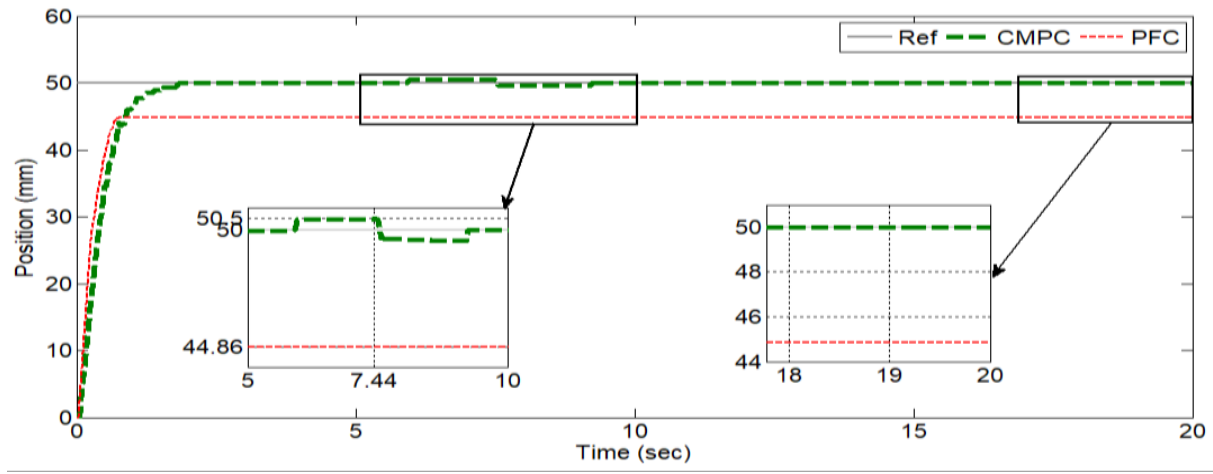

(a)

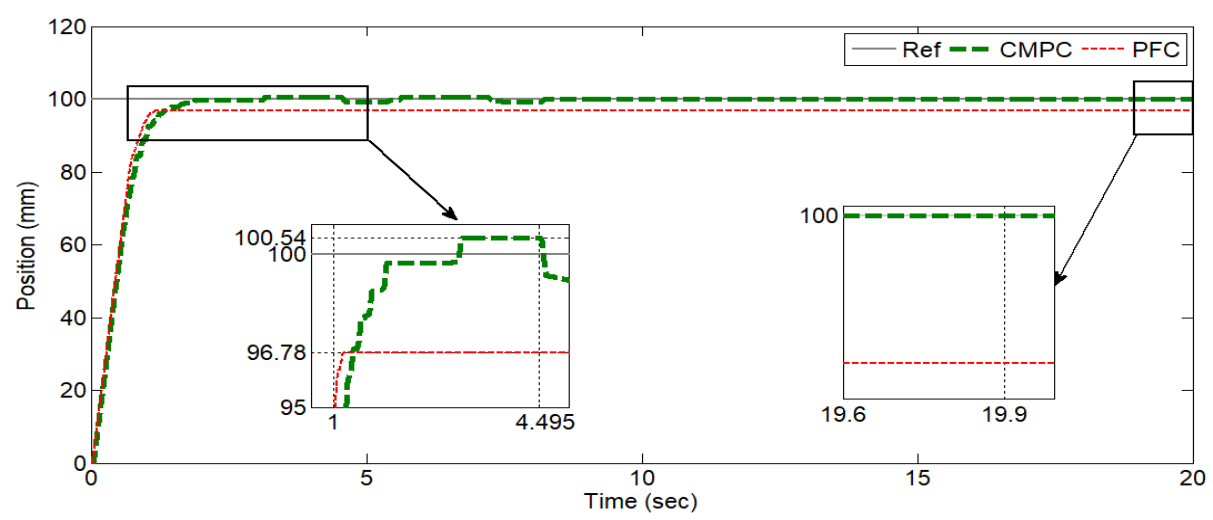

(b)

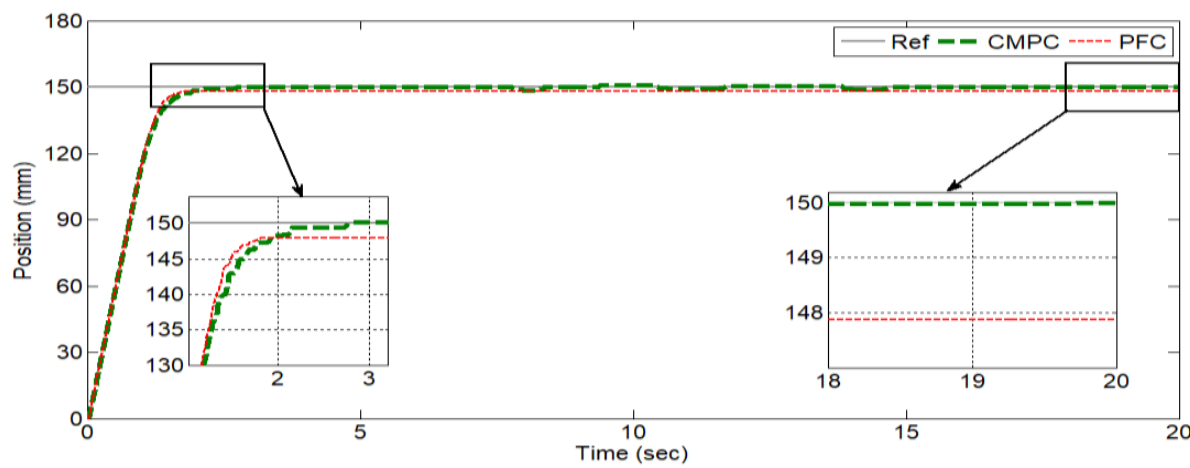

(c)

Figure 8. Experimental response when position distance is at; (a) $50 \mathrm{~mm}$, (b) $100 \mathrm{~mm}$, and (c) $150 \mathrm{~mm}$

Given the response of $O S$, NG-CMPC and PFC-O produced a response without $O S$ for all position distances. CMPC gave a system response with $O S$ of between $0.5 \%$ and $1 \%$, whereas PI produced significantly large $O S(>20 \%)$, for all position distances. The comparison of $O S$ performance between CMPC and NG-CMPC clearly showed that NG-CMPC consistently succeeded in improving the OS of the CMPC. Concerning accuracy, PFC-O was less accurate compared to the CMPC, NG-CMPC, and PI strategies. Although the increase in position distance reduced the $e_{s s}$ produced by PFC-O, however, the positioning control of pneumatic using PFC-O as a strategy was still not accurate. Furthermore, the $e_{s s}$ produced by PFC-O was still high, which were $5.140 \mathrm{~mm}, 3.220 \mathrm{~mm}$, and $2.120 \mathrm{~mm}$ for the position

Enhancement in pneumatic positioning system using nonlinear gain constrained... (Siti Fatimah Sulaiman) 
distances of $50 \mathrm{~mm}, 100 \mathrm{~mm}$, and $150 \mathrm{~mm}$, respectively. These are contrary to other strategies that gave almost $0 \mathrm{~mm}$ of $e_{s s}$ during the control operations.

Therefore, to summarise, the incorporation of $k_{n l}$ into the CMPC algorithm was considered as a novel control technique to improve the overall transient response performance, particularly in CMPC strategy. Uisng CMPC, accurate positioning control with a minimal overshoot of pneumatic positioning system can be provided, however fast speed response is very difficult to achieve. NG-CMPC improved the speed of the response and overshoot given by CMPC, with a small value of steady-state error. PFC-O produced an almost similar response of speed and overshoot as NG-CMPC, however, its steady-state error was substantial. The PI produced very large overshoot in the system response, with the penalty of increased settling time. The results obtained using NG-CMPC are satisfactory and show an improved transient response performance given by CMPC by providing fast speed response without overshoot, for all position distances.

Table 2. Summary of the experimental response using different control strategies for position distance of 50 $\mathrm{mm}, 100 \mathrm{~mm}$, and $150 \mathrm{~mm}$

\begin{tabular}{cccccc}
\hline \multirow{2}{*}{ Distance $(\mathrm{mm})$} & \multirow{2}{*}{ Transient performance } & CMPC & NG-CMPC & PFC-O & PI \\
\hline \multirow{4}{*}{50} & $t_{r}(\mathrm{~s})$ & 0.775 & 0.390 & 0.464 & 0.475 \\
& $t_{s}(\mathrm{~s})$ & 1.528 & 0.580 & 0.670 & 3.500 \\
& $O S(\%)$ & 1.000 & 0 & 0 & 21.504 \\
& $e_{s s}(\mathrm{~mm})$ & 0 & 0.020 & 5.140 & 0.010 \\
\multirow{3}{*}{100} & $t_{r}(\mathrm{~s})$ & 0.870 & 0.687 & 0.706 & 0.664 \\
& $t_{s}(\mathrm{~s})$ & 1.550 & 0.988 & 1.026 & 3.345 \\
& $O S(\%)$ & 0.540 & 0 & 0 & 38.467 \\
& $e_{s s}(\mathrm{~mm})$ & 0 & 0 & 3.220 & 0.190 \\
\multirow{3}{*}{150} & $t_{r}(\mathrm{~s})$ & 1.103 & 1.023 & 1.049 & 1.010 \\
& $t_{s}(\mathrm{~s})$ & 1.765 & 1.406 & 1.482 & 3.826 \\
& $O S(\%)$ & 0.547 & 0 & 0 & $\max$ \\
& $e_{s s}(\mathrm{~mm})$ & 0 & 0.050 & 2.120 & 0.310 \\
\hline
\end{tabular}

\section{CONCLUSION}

This study proposed a nonlinear gain constrained model predictive controller (NG-CMPC) as new control strategy to enhance pneumatic positioning system transient response while considering the constraints of system. In this study, the nonlinear gain function was incorporated within the conventional constrained MPC (CMPC) algorithm for the purpose of improving the speed response and accuracy of the pneumatic positioning system. At the end of this study, the performances of the proposed NG-CMPC to control the pneumatic positioning system were compared with $\mathrm{CMPC}$, proportional-integral (PI), and predictive functional control with observer (PFC-O). From the experimental results, it was found that NG-CMPC improved the performance of transient response given by CMPC by providing fast speed response with minimal/without overshoot. Meanwhile, comparison with PI and PFC-O revealed that the proposed NGCMPC gave a good improvement of speed and overshoot in the system response. NG-CMPC are capable to provide fast speed response without overshoot for all position distances and also succeeded in maintaining the steady-state accuracy in the pneumatic positioning system response regardless of whether the load varied. Therefore, showing that incorporating nonlinear gain function in the controller algorithm can improve the system transient response. Thus, all these findings proved that the linear controller remains relevant in controlling the position of the pneumatic system and certain modifications can improve the performance of system.

\section{ACKNOWLEDGEMENTS}

The authors would like to acknowledge Universiti Teknikal Malaysia Melaka (UTeM) and Ministry of Higher Education (MOHE) of Malaysia for their financial support. The authors would also like to express their gratitude to the individuals who were directly or indirectly involved in this study.

\section{REFERENCES}

[1] X. Li, K. Sun, C. Guo, T. Liu, and H. Liu, "Enhanced Static Modeling of Commercial Pneumatic Artificial Muscles," Assembly Automation, vol. 40, no. 3, pp. 407-417, 2020, doi:10.1108/AA-04-2019-0060.

[2] J. Ren, J. Zhong, L. Yao, and Z. Guan, "Experimental Investigation and Theoretical Modelling of a High-Pressure Pneumatic Catapult Considering Dynamic Leakage and Convection,” Entropy, vol. 22, no. 9, p. 1010, 2020, doi: 10.3390/e22091010. 
[3] S. N. S. Salim, M. F. Rahmat, L. Abdullah, S. A. Shamsudin, M. Zainon, and A. F. M. Amin, "Enhanced SelfRegulation Nonlinear PID for Industrial Pneumatic Actuator," International Journal of Electrical and Computer Engineering (IJECE), vol. 9, no. 4, pp. 3015, 2019, doi:10.11591/ijece.v9i4.pp3015-3024.

[4] R. Chang, B. Wang, and H. Ji, "Position control with pole placement for a miniature double-cylinder hydrostatic actuator," 2021 IEEE 19th International Power Electronics and Motion Control Conference (PEMC), 2021, pp. 486-493, doi: 10.1109/PEMC48073.2021.9432557.

[5] S. S. Ali, "Position Control of Ball and Beam System Using Robust Ho Loop Shaping Controller," Indonesian Journal of Electrical Engineering and Computer Science (IJEECS), vol. 19, no. 1, pp. 91-98, 2020, doi:10.11591/ijeecs.v19.i1.pp91-98.

[6] N. M. Tahir, M. Muhammad, B. B. Muhammad, H. Liman, A. Y. Zimit, and A. S. Tijjani, "Optimized OutputBased Input Shaping for Control of Single-Link Flexible Manipulator Using Linear Matrix Inequality,” Indonesian Journal of Electrical Engineering and Computer Science (IJEECS), vol. 20, no. 1, pp. 109-116, 2020, doi:10.11591/ijeecs.v20.i1.pp109-116.

[7] M. Chavoshian and M. Taghizadeh, "Recurrent Neuro-Fuzzy Model of Pneumatic Artificial Muscle Position," Journal of Mechanical Science and Technology, vol. 34, np. 1, pp. 499-508, 2020, doi: 10.1007/s12206-019-11548.

[8] H. Qi, G. M. Bone, and Y. Zhang, "Position Control of Pneumatic Actuators Using Three-Mode Discrete-Valued Model Predictive Control," Actuators, vol. 8, no. 3, p. 56, 2019, doi:10.3390/act8030056.

[9] Y. Zhang, X. Chen, H. Wang, and L. Zhang, "A Study on the Control of a Sensorless Pneumatic Joint Using Predictive Control Method," in IEEE Access, vol. 7, pp. 59923-59932, 2019, doi: 10.1109/ACCESS.2019.2913950.

[10] T. Alamirew, V. Balaji, and N. Gabbeye, "Comparison of PID Controller with Model Predictive Controller for Milk Pasteurization Process," Bulletin of Electrical Engineering and Informatics (BEEI), vol. 6, no. 1, pp. 24-35, 2017, doi: 10.11591/eei.v6i1.575.

[11] E. F. Camacho and C. B. Alba, Model Predictive Control (Advanced Textbooks in Control and Signal Processing), Springer-Verlag, 2004, doi: 10.1007/978-0-85729-398-5.

[12] S. A. Ajwad, M. I. Ullah, K. Baizid, and J. Iqbal, "A Comprehensive State-of-the-Art on Control of Industrial Articulated Robots," Journal of Balkan Tribological Association, vol. 20, no. 4, pp. 499-521, 2014.

[13] M. I. Ullah, S. A. Ajwad, M. Irfan, and J. Iqbal, "MPC and H-Infinity Based Feedback Control of Non-Linear Robotic Manipulator," 2016 International Conference on Frontiers of Information Technology (FIT), 2016, pp. 136-141, doi: 10.1109/FIT.2016.033 3.

[14] J. Iqbal, "Modern Control Laws for an Articulated Robotic Arm," Engineering, Technology \& Applied Science Research, vol. 9, no. 2, pp. 4057-4061, 2019, doi:10.48084/etasr.2598.

[15] Y. Wakasa, R. Sasaki, K. Tanaka, and T. Akashi, "Servo Control of Pneumatic Systems Considering Input and Output Constraints," 2007 IEEE International Conference on Control Applications, 2007, pp. 765-770, doi: 10.1109/CCA.2007.4389325.

[16] S. F. Sulaiman, et al., "Enhanced Position Control for Pneumatic System by Applying Constraints in MPC Algorithm," International Journal of Electrical and Computer Engineering (IJECE), vol. 7, no. 3, pp. 1633-1642, 2017, doi:10.11591/ijece.v7i3.pp1633-1642.

[17] S. F. Sulaiman, M. F. Rahmat, A. A. M. Faudzi, K. Osman and A. R. Azira, "Constrained MPC Based Laguerre Network to Control IPA Positioning System," in Proceedings of Mechanical Engineering Research Day 2018 (MERD 2018), 2018, pp. 46-47.

[18] J. Iqbal, M. Ullah, S. G. Khan, B. Khelifa, and S. Ćuković, "Nonlinear Control Systems- A Brief Overview of Historical and Recent Advances," Nonlinear Engineering, vol. 6, no. 4, pp. 301-312, 2017, doi:10.1515/nleng2016-0077.

[19] H. Seraji, "A New Class of Nonlinear PID Controllers for Robotic Applications," Journal of Robotic Systems, vol. 15, no. 3, pp. 161-181, 1998, doi:10.1016/S1474-6670(17)44245-5.

[20] M. F. Rahmat, Sy Najib Sy Salim, N. H. Sunar, A. 'A. M. Faudzi, Z. H. Ismail, and K. Huda, "Identification and Non-Linear Control Strategy for Industrial Pneumatic Actuator," International Journal of the Physical Sciences, vol. 7, no. 17, pp. 2565-2579, Apr 2012, doi:10.5897/ijps12.030.

[21] S. N. S. Salim, M. F. Rahmat, A. 'A. M. Faudzi, Z. H. Ismail, and N. Sunar, "Position Control of Pneumatic Actuator Using Self-Regulation Nonlinear PID," Mathematical Problems in Engineering, vol. 2014, pp. 1-12, June 2014, doi:10.1155/2014/957041.

[22] Y. X. Su, DongSun, and B. Y. Duan, "Design of an Enhanced Nonlinear PID Controller," Mechatronics, vol. 15, no. 8, pp. 1005-1024, March 2015, doi:10.1016/j.mechatronics.2005.03.003.

[23] L. Abdullah, et al., "Evaluation on Tracking Performance of PID, Gain Scheduling and Classical Cascade P/PI Controller on XY Table Ballscrew Drive System," World Applied Sciences Journal, vol. 21 (Special Issue of Engineering and Technology), pp. 1-10, 2013, doi:10.5829/idosi.wasj.2013.21.1001.

[24] S. I. Samsudin, M. F. Rahmat, and N. A. Wahab, "Nonlinear PI Control with Adaptive Interaction Algorithm for Multivariable Wastewater Treatment Process," Mathematical Problems in Engineering, vol. 2014, pp. 1-13, 2014, doi:10.1155/2014/475053.

[25] W. Alam, S. Ahmad, A. Mehmood, and J. Iqbal, "Robust Sliding Mode Control for Flexible Joint Robotic Manipulator via Disturbance Observer," Interdisciplinary Description of Complex Systems: INDECS, vol. 17, no. 1-B, pp. 85-97, 2019, doi:10.7906/indecs.17.1.11. 